\title{
THE SCOPE OF RADIOTHERAPY IN CARCINOMA OF THE BRONCHUS
}

\author{
By Gwen Hilton, D.M.R.E., F.F.R. \\ Director of the Radiotherapy Depariment, University College Hospital
}

It is not yet possible to compare the results of treatment of carcinoma of the bronchus by surgery alone with those by radiotherapy since no one has published the results of cases treated by radiotherapy which could have been dealt with by operation. A true comparison would necessitate a comparable series of cases. Surgery is still considered to be the treatment of choice, but unfortunately only a small proportion of cases are suitable for operation. When the patient is first seen the disease, in the majority of cases, is too widespread for a pneumonectomy to be done. Out of a series of $I, 028$ cases seen by Brock (1952) between 1941 and $195^{\circ}$ an attempt at resection was made in only 146 . All the inoperable cases are not, however, fit for radiotherapy. Many of them will not only derive no benefit from irradiation, but may actually be made worse by the treatment. In Tod's enquiry (I94I) into the extent to which cancer patients in Great Britain receive radiotherapy, it was estimated that 50 per cent. of the patients suffering from cancer of the lung require $\mathrm{X}$-ray treatment. Out of 360 cases seen by the author (1949), I47 were thought to be unfit for any form of X-ray treatment either radical or palliative. There must therefore be the same careful selection of cases for irradiation as there is for surgery. Recently a combination of surgery and post-operative radiotherapy has been used in some cases; for instance in those cases in which it was known that glands involved with growth were left behind after pneumonectomy. Pre-operative $\mathrm{X}$-ray treatment has been given to a few so-called ' inoperable' cases which are said to have become operable after irradiation, and a pneumonectomy has then been carried out. Cleland (195I) reports five cases which had been considered inoperable at bronchoscopy on account of the proximity of the growth to the trachea or else of widening below the carina. These cases were given $\mathrm{X}$-ray treatment and subsequently it became possible to remove the lung. Smithers (1953) also reports five cases treated with pre-operative irradiation and pneumonectomy. Two of these patients are alive over three years.
Unfortunately radical X-ray treatment, i.e. treat- $\vec{\omega}$ ment given with the intent to cure, can only be carried out in a small number of patients. Theyo must be in relatively good condition and the tumour must be limited in size. It should not be larger than about $8 \mathrm{~cm}$. in diameter. Radicalcu treatment is indicated also in patients who haveoo intra-bronchial tumours situated too close to the 9 carina for a pneumonectomy. It is also indicated $\overrightarrow{+}$ in patients with technically operable tumours in ? which the malignancy is known to be high or who either from age or some other cause cannot stand an operation.

Palliative X-ray treatment on the other hand one of the most useful weapons we have at the present time for relieving some of the distressipg $\vec{c}$ symptoms from which patients with carcinoma of the bronchus suffer. Many practitioners are sti unaware of the great benefit which can be derived from it, and only remember the reactions produced in the early days of radiotherapy. Palliative treat-o ment if correctly given should cause no severeo local reaction or irradiation sickness. It is in- $\varrho$ dicated in patients in whom the cancer is limited in $\overrightarrow{\vec{O}}$ extent but whose general condition would not 3 stand the high dose of radical treatment, and in? patients with an extensive carcinoma but in good? general condition, who show one or more of the following signs or symptoms. Superior vena caval $\underline{3}$. obstruction; the relief is often dramatic and is one of the most useful clinical effects. Pressure on the oesophagus by enlarged mediastinal glands causing응 dysphagia; if this is relieved the patient may be saved from a gastrostomy. Haemoptysis; this is almost always stopped, although the effect may $>$ only be temporary. Cough is often diminished and을. sometimes completely stopped and dyspnoea may be relieved. Pain is relieved if it is due to $a^{\text {G }}$ secondary deposit. Neuritic symptoms and painful ${ }^{N}$ pulmonary arthropathy, which are sometimes ${ }_{\omega}^{N}$ associated with carcinoma of the bronchus mayo subside after irradiation of the primary cancer ino the bronchus. But pain is rarely relieved if it is in the chest and due to direct transpleural invasion? or to a tumour arising in the superior sulcus. 
Symptoms of raised intracranial pressure may be completely relieved if they are due to a cerebral metastasis, particularly of the oat cell or undifferentiated type of carcinoma.

Some patients will not benefit even from palliative X-ray treatment. If they are ill and wasted and if they have pulmonary infection and a raised pulse rate they will not stand a dose of $\mathrm{X}$-rays high enough to be of any value. If the tumour is very large, involving a whole lobe or a whole lung it is impossible to give an adequate dose to the entire tumour-bearing area without causing too great a constitutional disturbance. A large effusion, either serous or purulent scatters the $\mathrm{X}$-rays and prevents an adequate dose reaching the tumour. Aspiration is useless since the fluid reaccumulates so quickly. Nor can an adequate dose be given if there is extensive pleural involvement even if there is no effusion. Radioactive colloidal gold has been introduced into the pleural cavity in some of these cases in an attempt to irradiate the growth in the pleura, but this work is still in the experimental stage and only a small number of patients have so far been treated in this way. X-rays cannot be applied to a septic lung without the risk of necrosis perhaps followed by fatal haemorrhage. If, however, the sepsis can be relieved by bronchoscopic drainage and/or appropriate antibiotics, then irradiation carefully given can be successful. X-rays cannot be applied to a lung in the presence of active tuberculosis since irradiation may be followed by a rapid spread of the tuberculous process. Often an apparently inactive focus may be lighted up by irradiation.

It is usually easy to decide whether the patient should have either radical or palliative irradiation or whether he is unfit for X-ray treatment, but sometimes the decision is extremely difficult. Only a highly developed clinical sense and long experience will enable the radiotherapist to make the right decision. The patient should be admitted to the hospital for the treatment if possible, especially during the winter months, and everything should be done to improve the general condition and increase the percentage of haemoglobin. A blood transfusion or intravenous iron may be necessary before the course of treatment is started if the level of haemoglobin is very low. Sepsis if present should be deal, with. Suitable antibiotics can often control the infection and thus many more patients can be treated nowadays than was formerly possible. When bronchoscopy is performed an attempt should be made to remove as much pus as possible and postural drainage should be practised.

The extent of the primary growth together with that of the glandular involvement should be estimated as far as possible before deciding whether radical or palliative treatment should be given. One of the chief causes of failure in the $\mathrm{X}$-ray treatment of carcinoma of the bronchus is insufficient dosage to the whole of the cancerous tissue owing to lack of knowledge of its full extent. The whole range of radiological methods should be employed to assess this before planning the fields to be irradiated. On account of post-irradiation changes which frequently occur in normal lung tissue after a high dose of X-rays, irradiation should be confined as far as possible to the diseased lung. Rotation therapy or multiple small field therapy with beam direction is used if radical treatment is to be given, but only two large fields, one anterior and one posterior are needed if the treatment is palliative. The radical course of treatment lasts six or seven weeks, but the palliative dose should be sufficient to relieve the more serious symptoms, and is kept as low as possible and given as quickly as the patient will tolerate it. Fauvet and Huguenin (I 949) state that the results of irradiation of cancer of the bronchus have been improved by giving atropine sulphate in doses of one two-hundredth of a grain. Atropine is thought to work not only by diminishing secretion but also by relaxing bronchial spasm, thus facilitating drainage. Antibiotics should be given during the course as long as sepsis is present. Cough and pain should be treated with sedatives to enable the patient to get as much rest as possible. In caseso which respond to $\mathrm{X}$-ray treatment, the general condition should begin to improve after 10 to 14 days' treatment. The weight should increase throughout the course and for many weeks after the treatment is finished. The failure to put on some weight despite an improvement in chest symptoms and in the radiological appearance is nearly. always an indication of metastases outside the thorax.

Serial X-rays of the chest should be taken at monthly intervals after the treatment is over in order to assess the full result of irradiation. The response may be seen within a few days in cases of oat cell or undifferentiated carcinoma of the bronchus, and the opacity caused by the tumour may have disappeared by the end of the course but the response in cases of adenocarcinoma and of squamous cell carcinoma is much slower and the full effect may not be seen for some months. A second course of X-ray treatment should never be given as long as radiological improvement continues. It is unwise to make a bronchoscopic examination within six weeks following irradiation as the reaction in the mucosa of the bronchus will not have subsided. As well as the diminution in the size of the opacities due to the response of the growth to irradiation, fresh shadows may appear in the lung fields which are due to post-irradiation 
changes in the normal lung tissue. It is of great importance to be able to recognize these changes since they may be taken for a recurrence. If this mistake is made and more $\mathrm{X}$-ray treatment given, necrosis and abscess of the lung are likely to follow the second course of irradiation. In a number of patients there are no symptoms or signs, despite marked radiological changes in the lung fields. In others the pulse rate is raised and there is dyspnoea on exertion with paroxysmal attacks of coughing, pain is rare; some begin with signs of an acute pulmonary infection with slight fever and mucopurulent sputum which is diagnosed as influenza or pneumonia. Occasionally there are frequent small haemoptyses or a sero-fibrinous pleurisy with a lymphocytic count in the fluid. The signs and symptoms are usually of short duration but occasionally the syndrome is protracted. The average time of appearance of these radiological changes is four months, but sometimes they appear near the end of treatment or they may be delayed for as long as 18 months after irradiation. Diagnosis is often extremely difficult on account of the variability of the clinical picture and of the physical signs and it depends largely on the interpretation of the X-ray picture.

It is not possible to assess the effect of radiotherapy on survival since the duration of life in untreated cases of carcinoma of the bronchus is so variable. A few patients live for a number of years after radiotherapy but some untreated cases have also been reported to survive for as long as six to seven years after the diagnosis had been made. The average duration of life of patients with an oat-cell or undifferentiated carcinoma is. less than those with a squamous or an adeno carcinoma. In the author's series of cases proved? histologically which had received radical X-raye treatment, the average duration of life of those with an oat-cell or undifferentiated carcinoma wass I 4 months, with a squamous carcinoma 18 month and with an adenocarcinoma 28 months. It hass yet to be seen whether pre-operative irradiation will render more cases operable. The value of palliative X-ray therapy if properly given is un $\vec{\omega}$ doubted. Cough, dyspnoea, haemoptyses and superior vena caval obstruction can often bह alleviated. The general condition improves if these symptoms are relieved, and the patient mayi enjoy some months of normality. A number of patients become well enough to return to work foß a time. The final illness is usually short and deatlos may be fairly sudden from haemorrhage or pneumonia.

\section{BIBLIOGRAPHY}

BROCK (1952), Personal communication.

CLELAND, W. P. (195I) "The Management of the Patient wit Inoperable Cancer of the Chest,' 7. Thor. Surg., 22, No. 5, 5013

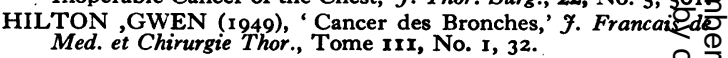

HUGUENIN, R., and FAUVET, J. (1949), 'Cents Canœrs. Bronchiques traités par Radiothérapie,' $¥$. Francais de Medo Chirurgie Thor., Tome III, p. 6 I.

SMITHERS, D. W. (1953), 'Facts and Fancies about Cances. the Lung,' Brit. med. F., No. 4822 , 1235.

TOD, M. (194I), 'An Inquiry into the extent to which CanceD Patients in Great Britain receive Radiotherapy, John Sherrat and Son, Altrincham.

\title{
SURGERY OF CARCINOMA OF THE BRONCHUS
}

\author{
By L. L. Bromley, M.ChIR., F.R.C.S. \\ Surgeon in Charge of the Thoracic Department, St. Mary's Hospital.
}

Carcinoma of the bronchus is a most lethal form of malignant disease. All too frequently patients present for treatment with the condition hopelessly advanced. It is probably fair to say that not more than one patient out of every four seen by a surgeon is likely to be suitable for a thoracotomy. The results in patients who do have a resection of the lung and its contained tumour are not favourable; the results can be compared with those of resection of the stomach or rectum.
One has not to look far to find the chief reason for the deadliness of carcinoma of the bronchuser Not only is the lung very richly supplied with lymphatics but it is also an extremely vascular w organ; it can be the simplest matter for cancero cells multiplying in the neighbourhood of pulmonary capillaries to enter the circulation, slip away through the heart and settle down at the endes of a systemic arteriole anywhere in the body. 0 Macroscopic growth of tumour in the pulmonaryo 